\title{
Entanglement and squeezing in a two-mode system: theory and experiment
}

\author{
V. Josse, A. Dantan, A. Bramati and E. Giacobino \\ Laboratoire Kastler Brossel, Université Pierre et Marie Curie, 4 place Jussieu, \\ F75252 Paris Cedex 05, France
}

\begin{abstract}
We report on the generation of non separable beams produced via the interaction of a linearly polarized beam with a cloud of cold cesium atoms placed in an optical cavity. We convert the squeezing of the two linear polarization modes into quadrature entanglement and show how to find out the best entanglement generated in a two-mode system using the inseparability criterion for continuous variable [Duan et al., Phys. Rev. Lett. 84, 2722 (2000)]. We verify this method experimentally with a direct measurement of the inseparability using two homodyne detections. We then map this entanglement into a polarization basis and achieve polarization entanglement.

PACS numbers: 42.50.Lc, 42.65.Pc, 42.50.Dv
\end{abstract}

\section{Introduction}

With the recent progress in the quantum information field, there has been a lot of interest in entanglement in the continuous variable (CV) regime. Criteria to demonstrate and quantify CV entanglement have been developed [1, 2] and experimentally tested 3, 4, 5, 6, 7, 8. In particular, the possibility to map a quantum polarization state of light onto an atomic ensemble [9] has stirred a great deal of attention to the quantum features of polarized bright beams. The notion of polarization entanglement, i.e. entanglement between Stokes parameters of two spatially separated beams, has been investigated by Korolkova et al. [10] and first demonstrated by Bowen et al. [7] by mixing two independent squeezed beams produced by OPAs. Polarization entanglement was also achieved via the Kerr non linearity of optical fibers [11] and cold atoms [8]. These experiments are important steps in connection with quantum teleportation [12, quantum dense coding [13, entanglement swapping [14] and, more generally, characterizing entanglement in the CV regime [15].

In this paper, we report on the generation of non separable beams via the interaction of a linearly polarized light beam with a cloud of cold cesium atoms placed in an optical cavity [8]. In previous works [16, 17, we have shown that, after the non linear interaction with the atoms, two modes of the light exiting the cavity were squeezed: the mean field mode, but also the orthogonally polarized vacuum. We develop here a general method to find out the best entanglement - as measured with the inseparability criterion [2] - 
produced in a two mode system and characterize the correlation properties of the system in the Poincaré sphere. The main result is that the maximal entanglement corresponds to the sum of the minimum noises of two "uncorrelated" modes. The maximally entangled modes are then circularly polarized with respect to these modes. We stress the similarity with the usual entanglement experiments, which mix independent squeezed beams on a beamsplitter [7, 11. Moreover, we show that this mixing of two independent beams is equivalent to rotating the polarization basis of a single beam exhibiting correlations between polarization modes. This intuitive approach could be of interest for the study of systems in which quantum correlations exist between polarization modes, and allows one to think in terms of independent beams mixing.

We then apply these results to our experiments [8], show experimental evidence of both quadrature entanglement and polarization entanglement. In Sec. 3 quadrature entanglement is demonstrated by figuring out the maximally entangled modes and checking the inseparability criterion between these modes in a direct detection scheme. In Sec. 4 we map the entanglement into a polarization basis via the mixing of our quadrature entangled modes with an intense coherent beam, the phase of which is locked to that of the first beam. We therefore measure directly the Stokes parameters fluctuations of the two spatially separated beams, thus demonstrating polarization entanglement.

\section{Looking for maximal entanglement}

\subsection{General method}

In this section we develop a general method to find out the maximal entanglement in a two-mode system. We start with a "black box" - in our case the atomic medium in the cavity - out of which comes a light beam with unknown quantum properties. Let us stress that the goal of this Section is to develop a method to characterize quantum properties, such as entanglement and squeezing, which have been previously created between some polarization modes by some interaction. Let us denote by $A_{a}$ and $A_{b}$ two orthogonally polarized modes of this beam. They satisfy the standard bosonic commutation relations $\left[A_{\alpha}, A_{\beta}^{\dagger}\right]=\delta_{\alpha \beta}$. The usual quadrature operators, with angle $\theta$ in the Fresnel representation,

$$
X_{\alpha}(\theta)=A_{\alpha} e^{-i \theta}+A_{\alpha}^{\dagger} e^{i \theta}, \quad Y_{\alpha}(\theta)=X_{\alpha}(\theta+\pi / 2) \quad(\alpha=a, b)
$$

are the continuous variable analogous of the EPR-type operators as introduced by Einstein, Podolsky and Rosen [18. The criterion derived by Duan et al. and Simon [2] sets a limit for inseparability on the sum of these EPR-type operators variances

$$
\mathcal{I}_{a, b}(\theta)=\frac{1}{2}\left[\delta\left(X_{a}+X_{b}\right)^{2}(\theta)+\delta\left(Y_{a}-Y_{b}\right)^{2}(\theta)\right]<2
$$

For Gaussian states, $\mathcal{I}_{a, b}(\theta)<2$ is a sufficient condition for entanglement and

has already been used several times to quantify continuous variable entanglement 
[10, 12, 11, 8]. In this Section we look for the best entanglement produced in the system: using unitary transformations we therefore seek to minimize $\mathcal{I}_{a, b}$ with respect to $a, b$ and $\theta$. Expanding (11), one gets

$$
\mathcal{I}_{a, b}(\theta)=\left\langle\delta A_{a}^{\dagger} \delta A_{a}+\delta A_{a} \delta A_{a}^{\dagger}+\delta A_{b}^{\dagger} \delta A_{b}+\delta A_{b} \delta A_{b}^{\dagger}\right\rangle+4\left|\left\langle\delta A_{a} \delta A_{b}\right\rangle\right| \cos \left[2\left(\theta-\theta_{a, b}\right)\right](2)
$$

where $\theta_{a, b}$ is the phase of $\left\langle\delta A_{a} \delta A_{b}\right\rangle$. The minimum value is reached for $\theta=\theta_{a, b} \pm \pi / 2$ :

$$
\mathcal{I}_{a, b}=\min _{\theta} \mathcal{I}_{a, b}(\theta)=\left\langle\delta A_{a}^{\dagger} \delta A_{a}+\delta A_{a} \delta A_{a}^{\dagger}+\delta A_{b}^{\dagger} \delta A_{b}+\delta A_{b} \delta A_{b}^{\dagger}\right\rangle-4\left|\left\langle\delta A_{a} \delta A_{b}\right\rangle\right|(3)
$$

$\mathcal{I}_{a, b}$ does not depend on local unitary operations performed separately on $a, b$. It thus provides a good measurement of the entanglement between modes $a$ and $b$ and will be used throughout this paper. Consequently, one has to look for the polarization basis $\left(a^{*}, b^{*}\right)$ of the "maximally entangled modes" which minimizes $\mathcal{I}_{a, b}$. It is easy to see that the first term in (3) is independent of the polarization basis, since it is the trace of the correlation matrix of modes $a$ and $b$. The entanglement between $a$ and $b$ is therefore completely determined by the correlation term $\left|\left\langle\delta A_{a} \delta A_{b}\right\rangle\right|$.

In order to find the strongest correlations we turn to a particular basis for the fluctuations (we are only interested here in what happens to the "noise ellipsoid", regardless of the mean field): as shown in the Appendix A, there always exists two orthogonally polarized modes $A_{u}$ and $A_{v}$ such that $\left\langle\delta A_{u} \delta A_{v}\right\rangle=0$. The $u, v$ modes are "uncorrelated" in the sense of the inseparability criterion and satisfy $\mathcal{I}_{u, v}=\max _{a, b} \mathcal{I}_{a, b} \geq$ 2. Note that these modes are not uncorrelated stricto sensu, since $\left\langle\delta A_{u} \delta A_{v}^{\dagger}\right\rangle$ can be non zero. Moreover, our choice is not unique, since any $A_{u}^{\prime}=e^{i \theta_{u}} A_{u}$ and $A_{v}^{\prime}=e^{i \theta_{v}} A_{v}$ also satisfy the same property. To unambiguously determine the "uncorrelated" basis we choose modes $u$ and $v$ such that $\left\langle\delta A_{u}^{2}\right\rangle$ and $\left\langle\delta A_{v}^{2}\right\rangle$ are positive numbers. Physically, it means that we choose $u$ and $v$ such that their noise is minimum for the same quadrature $Y$.

Two orthogonally polarized modes $a$ and $b$ decompose on such a basis

$$
\begin{aligned}
& A_{a}=\beta A_{u}-\alpha e^{i \phi} A_{v} \\
& A_{b}=\alpha A_{u}+\beta e^{i \phi} A_{v}
\end{aligned}
$$

with $\alpha, \beta$ positive real numbers such that $\alpha^{2}+\beta^{2}=1$. The correlation term reads

$$
\left|\left\langle\delta A_{a} \delta A_{b}\right\rangle\right|^{2}=\alpha^{2} \beta^{2}\left[\left\langle\delta A_{u}^{2}\right\rangle^{2}+\left\langle\delta A_{v}^{2}\right\rangle^{2}-2\left\langle\delta A_{u}^{2}\right\rangle\left\langle\delta A_{v}^{2}\right\rangle \cos 2 \phi\right]
$$

and is maximal for $\phi=\pi / 2[\pi]$ and $\alpha=\beta=1 / \sqrt{2}$. The maximally entangled modes are then the circularly polarized modes with respect to modes $u, v$ :

$$
\begin{aligned}
& A_{a^{*}}=\frac{1}{\sqrt{2}}\left(A_{u}-i A_{v}\right) \\
& A_{b^{*}}=\frac{1}{\sqrt{2}}\left(A_{u}+i A_{v}\right)
\end{aligned}
$$


which satisfy

$$
\left|\left\langle\delta A_{a^{*}} \delta A_{b^{*}}\right\rangle\right|=\max _{a, b}\left|\left\langle\delta A_{a} \delta A_{b}\right\rangle\right|=\frac{1}{2}\left[\left\langle\delta A_{u}^{2}\right\rangle+\left\langle\delta A_{v}^{2}\right\rangle\right]
$$

Plugging this result in (31) and using (17/8), we obtain the maximal entanglement value as the sum of the minimal noise of the "uncorrelated" modes

$$
\mathcal{I}_{a^{*}, b^{*}} \equiv \min _{a, b} \mathcal{I}_{a, b}=\left\langle\delta X_{u}^{2}\right\rangle_{\min }+\left\langle\delta X_{v}^{2}\right\rangle_{\min }
$$

This is the main result of this Section: the best entanglement in the system is found between the circularly polarized modes in the $(u, v)$ basis, and it is equal to the sum of the $u, v$ mode minimal noises. This result stresses the link between entanglement and squeezing; associating to each polarization basis $(a, b)$ the sum of the minimal noises

$$
\begin{aligned}
\Sigma_{a, b} & \equiv\left\langle\delta X_{a}^{2}\right\rangle_{\min }+\left\langle\delta X_{b}^{2}\right\rangle_{\min } \\
& =\left\langle\delta A_{a}^{\dagger} \delta A_{a}+\delta A_{a} \delta A_{a}^{\dagger}+\delta A_{b}^{\dagger} \delta A_{b}+\delta A_{b} \delta A_{b}^{\dagger}\right\rangle-2\left[\left|\left\langle\delta A_{a}^{2}\right\rangle\right|+\left|\left\langle\delta A_{b}^{2}\right\rangle\right|\right]
\end{aligned}
$$

Eq. (9) clearly shows that looking for maximal entanglement is equivalent to looking for maximal squeezing produced by the system

$$
\mathcal{I}_{a^{*}, b^{*}}=\min _{a, b}\left[\left\langle\delta X_{a}^{2}\right\rangle_{\min }+\left\langle\delta X_{b}^{2}\right\rangle_{\min }\right] \equiv \Sigma_{\min }
$$

\subsection{Correlations in the Poincaré sphere}

A standard representation for the polarization state of light is provided by the Poincaré sphere [10], which relies on the Stokes parameters [20]. Given the special role played by the "uncorrelated" basis, we define the Stokes parameters from the $u, v$ modes

$$
\begin{array}{ll}
S_{0}^{\prime}=A_{u}^{*} A_{u}+A_{v}^{*} A_{v} & S_{1}^{\prime}=A_{u}^{*} A_{u}-A_{v}^{*} A_{v} \\
S_{2}^{\prime}=A_{u}^{*} A_{v}+A_{v}^{*} A_{u} & S_{3}^{\prime}=i\left(A_{v}^{*} A_{u}-A_{u}^{*} A_{v}\right)
\end{array}
$$

and we study the evolution of entanglement and squeezing when the polarization basis is rotated, that is, when the polarization state vector moves along the Poincaré sphere. In the general case the correlation properties of the system can be summarized as follows (see Appendix B for the demonstration of these results):

(i) Along the "uncorrelated" modes axis $S_{1}^{\prime}, \mathcal{I}_{a, b}$ is maximal by construction $\left(\left\langle\delta A_{u} \delta A_{v}\right\rangle=0\right)$ and these modes are never entangled

$$
\begin{aligned}
\mathcal{I}_{u, v} & =\max _{a, b} \mathcal{I}_{a, b}=\left\langle\delta A_{u}^{\dagger} \delta A_{u}+\delta A_{u} \delta A_{u}^{\dagger}+\delta A_{v}^{\dagger} \delta A_{v}+\delta A_{v} \delta A_{v}^{\dagger}\right\rangle \\
& =\left\langle\delta X_{u}^{2}\right\rangle+\left\langle\delta Y_{u}^{2}\right\rangle+\left\langle\delta X_{v}^{2}\right\rangle+\left\langle\delta Y_{v}^{2}\right\rangle \geq 2
\end{aligned}
$$


A

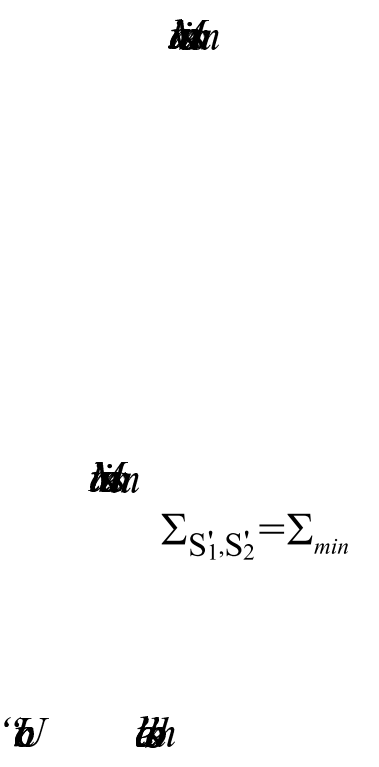

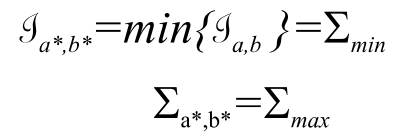

(c)
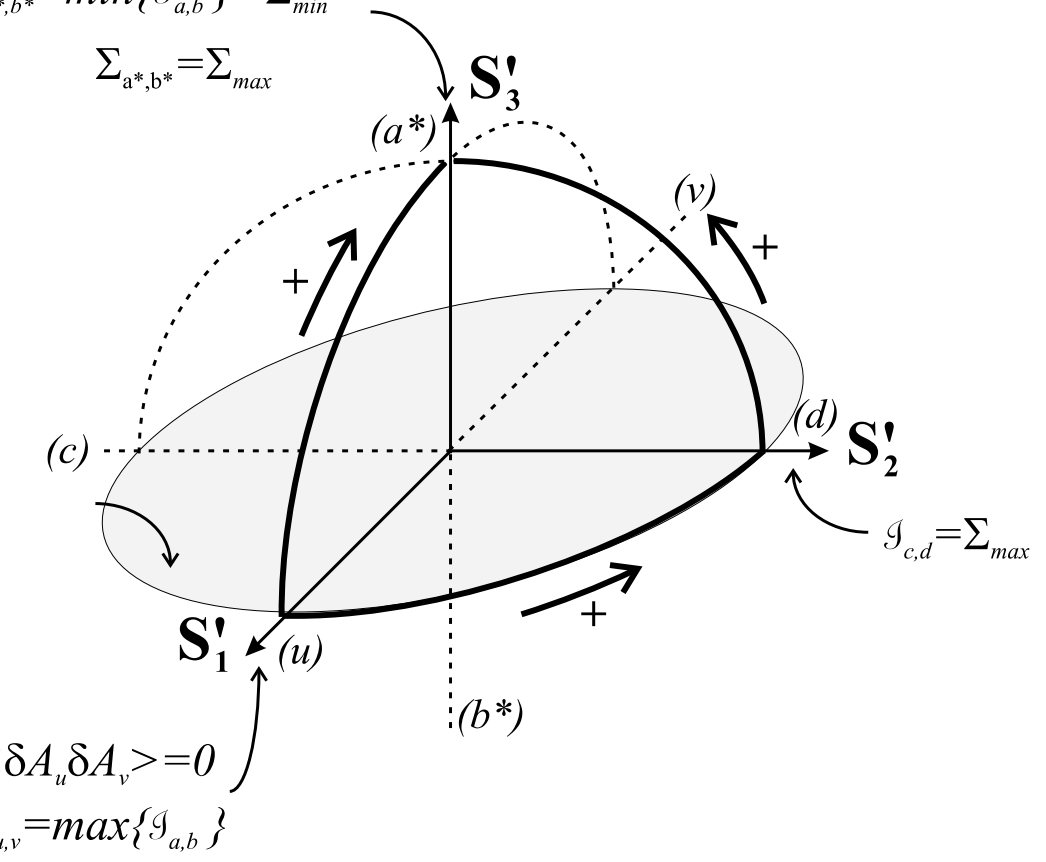

Figure 1. Quantum properties of the beam in the Poincaré sphere. The arrows with sign "+" correspond to increasing correlations.

These modes are characterized by the fact that $\mathcal{I}_{u, v}(\theta)$ is independent of $\theta$. The least noisy quadratures are the same and the noise reduction is maximal

$$
\Sigma_{u, v}=\Sigma_{\min }=\left\langle\delta X_{u}^{2}\right\rangle_{\min }+\left\langle\delta X_{v}^{2}\right\rangle_{\min }
$$

(ii) In the equatorial plane $\left(S_{1}^{\prime}, S_{2}^{\prime}\right)$, corresponding to the linearly polarized modes with respect to $u, v$, the noise reduction is also maximal and equal to $\Sigma_{\min }$. However, the entanglement is not constant: the best entanglement is obtained along the $S_{2}^{\prime}$ axis (modes at $45^{\circ}$ to the $u, v$ modes) and its value is equal to the weakest noise reduction $\Sigma_{\max }$

$$
\mathcal{I}_{c, d}=\Sigma_{\max }=\min \left[\left\langle\delta X_{u}^{2}\right\rangle_{\min }+\left\langle\delta X_{v}^{2}\right\rangle_{\max },\left\langle\delta X_{u}^{2}\right\rangle_{\max }+\left\langle\delta X_{v}^{2}\right\rangle_{\min }\right]
$$

(iii) Along the $S_{3}^{\prime}$ axis are the maximally entangled modes $a^{*}, b^{*}$, for which the entanglement is maximal (and equal to the best noise reduction value)

$$
\mathcal{I}_{a^{*}, b^{*}}=\min _{a, b} \mathcal{I}_{a, b}=\Sigma_{m i n}=\left\langle\delta X_{u}^{2}\right\rangle_{\min }+\left\langle\delta X_{v}^{2}\right\rangle_{\min }
$$

and for which the excess noise is the largest

$$
\Sigma_{a^{*}, b^{*}}=\left\langle\delta X_{a^{*}}^{2}\right\rangle_{\min }+\left\langle\delta X_{b^{*}}^{2}\right\rangle_{\min }=\Sigma_{\max }
$$


A graphical representation of these results is given in Fig. 1] at the poles there is maximal entanglement $\left(\mathcal{I}_{a^{*}, b^{*}}=\Sigma_{\min }\right)$ and the worst noise reduction $\left(\Sigma_{a^{*}, b^{*}}=\Sigma_{\max }\right)$. In the equatorial plane the noise reduction is optimal $\left(\Sigma_{S_{1}^{\prime}, S_{2}^{\prime}}=\Sigma_{\text {min }}\right)$. Note that the $c, d$ modes at " $\pm 45^{\circ}$ may be entangled while having optimal noise reduction.

\subsection{Interpretation}

An interpretation of the previous results can be given in connection with typical experiments in which entanglement is obtained by having independent beams interfere with each other [7, 11. Indeed, going from the $(u, v)$ to the $(a, b)$ basis with parameters $\alpha, \beta$ and $\phi$ [Eqs. (45)] is equivalent to combining the $u, v$ modes on a beamsplitter with transmission $T=\beta^{2}$, the $v$ mode being dephased by $\phi$ [see Fig. 2(a)]. Although, again, the $u, v$ modes are not completely uncorrelated, we can interpret the general results enunciated above as the result of an interference between two independent beams. This configuration is the one typically used to generate entangled beams: two squeezed beams on the same quadrature are produced separately - for instance with two OPAs [7, 15], or by using the Kerr-type non-linearity of optical fibers [11] - and then combined on a beamsplitter.

The $u, v$ modes are squeezed for the same quadrature $Y$, so that, in case of a zerodephasing before the beamsplitter, the squeezed and noisy quadratures do not mix and the noise reduction sum is conserved [Fig 2(b)]. The outgoing beams are linearly polarized with respect to the incoming ones $(\phi=0)$ and we retrieve the property (ii)

$$
\Sigma_{S_{1}^{\prime}, S_{2}^{\prime}}=\left\langle\delta X_{u}^{2}\right\rangle_{\min }+\left\langle\delta X_{v}^{2}\right\rangle_{\min }=\Sigma_{\min }
$$

If the dephasing before mixing is now equal to $\pi / 2$, the outgoing beams have excess noise on both quadratures [see Fig. 2(c)]. However, these noises are correlated and, obviously, all the more so for a 50/50 beamsplitter, since it maximally mixes the $u, v$ modes. This transformation is equivalent to going from the linearly polarized basis $(u, v)$ to the circularly polarized basis $\left(a^{*}, b^{*}\right)$, which is naturally the correlated basis

$$
\begin{aligned}
& \frac{1}{2}\left\langle\delta\left(X_{a^{*}}+X_{b^{*}}\right)^{2}(\theta)\right\rangle=\left\langle\delta X_{u}^{2}(\theta)\right\rangle<1 \quad \text { for } \theta=\frac{\pi}{2} \\
& \frac{1}{2}\left\langle\delta\left(Y_{a^{*}}-Y_{b^{*}}\right)^{2}(\theta)\right\rangle=\left\langle\delta X_{v}^{2}(\theta)\right\rangle<1 \quad \text { for } \theta=\frac{\pi}{2} \\
\text { Thus } \quad & \mathcal{I}_{a^{*}, b^{*}}=\left\langle\delta X_{u}^{2}\right\rangle_{\text {min }}+\left\langle\delta X_{v}^{2}\right\rangle_{\text {min }}<2
\end{aligned}
$$

When the $u, v$ modes are symmetrical (same noise properties), it can be shown that they are completely independent (see Sec. 3 for an example). The $a^{*}, b^{*}$ modes fluctuations are then the same for all quadratures.

Before applying these results to our experiment, we would like to emphasize that this analogy between the quantum properties of any system and those produced via 

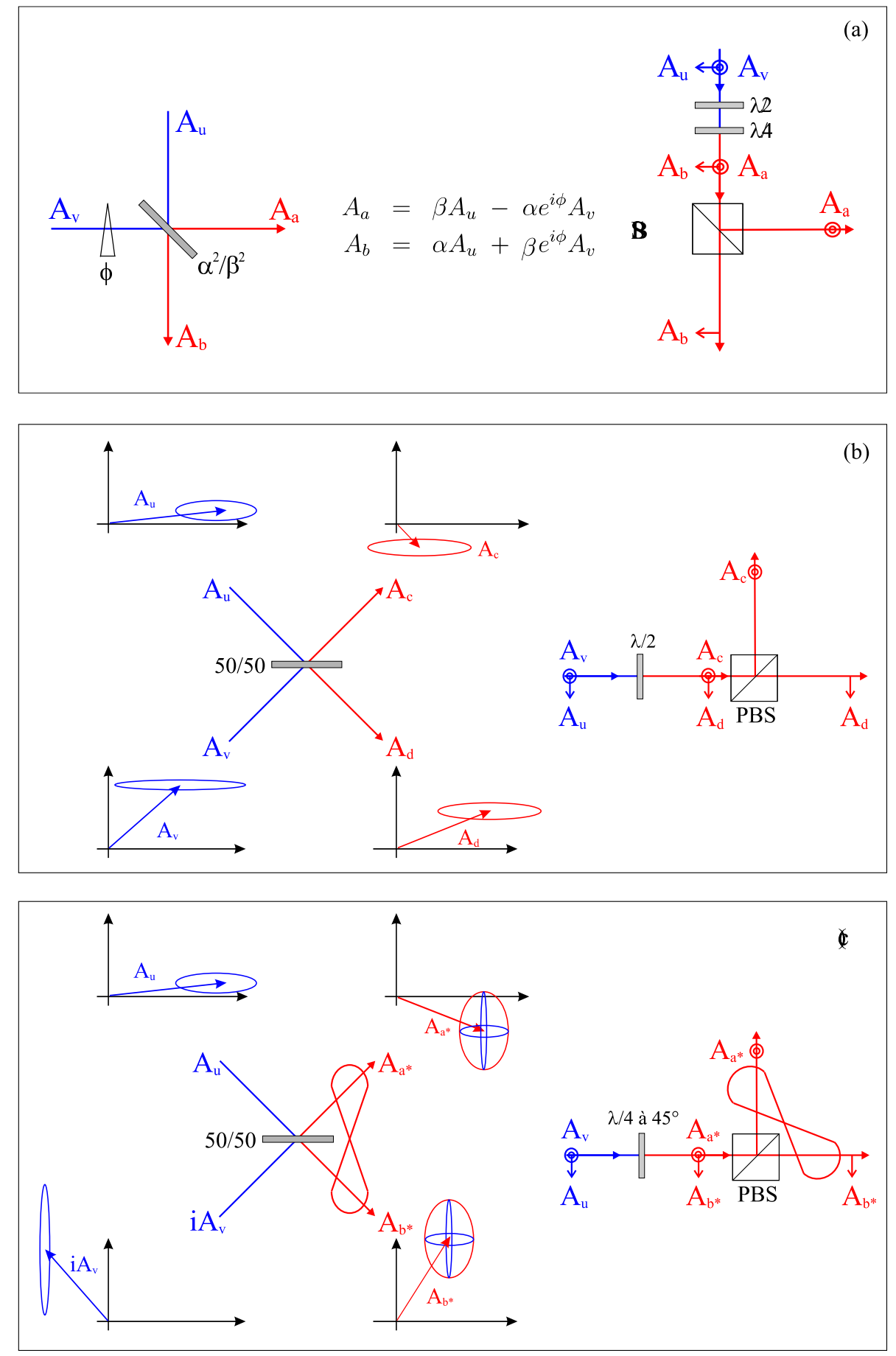

Figure 2. Interpretation of the correlations. (a) Equivalence between the transformations corresponding to the transmission by a beamsplitter (left) and a polarization basis rotation (right). (b) Squeezing conservation for the linearly polarized modes when there is no dephasing between the squeezed quadratures. (c) Generation of entangled modes via the interference of two modes squeezed for orthogonal quadratures.

the mixing of two independent beams provides us with a simple interpretation of the main results. This analysis is of particular interest to the study of systems for which 
the correlations are simultaneously produced inside a "black box". One has to find the "uncorrelated" modes $u, v$, an experimental signature being that the entanglement value $\mathcal{I}_{u, v}(\theta)$ does not depend on $\theta$. Once this basis is obtained, one can apply the previous formalism or, equivalently, think in terms of independent beams combination. This method allows, for instance, to determine the maximal correlations produced by an OPO inside which are inserted birefringent elements [19.

\section{Quadrature entanglement}

In our experiment [17, the black box consists in an optical cavity containing a cloud of cold cesium atoms into which is sent an off-resonant light beam. In this Section we first present the principle of a detection scheme allowing for a direct measurement of the quadrature entanglement given by (11). We then study the entanglement generated in the case of a linear incident polarization, which is qualitatively different from the circular polarization case.

\subsection{Entanglement measurement principle}

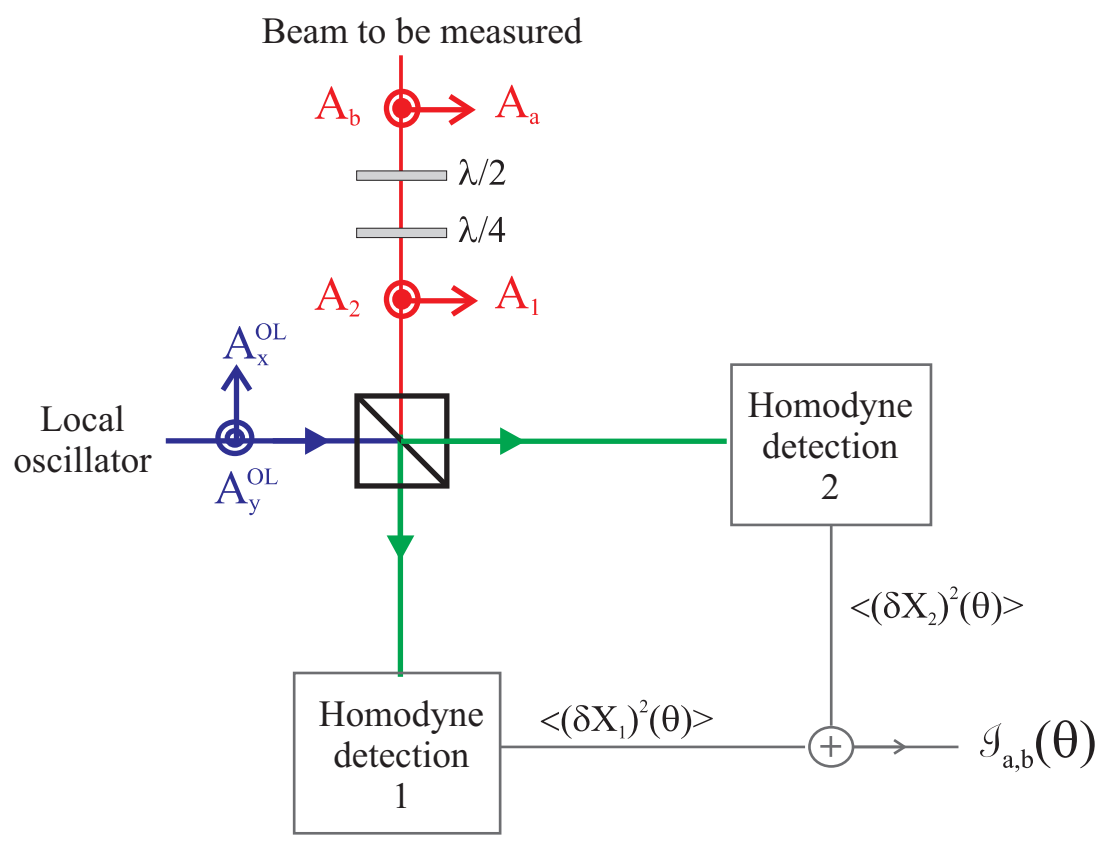

Figure 3. Schematic of the $a, b$ modes entanglement measurement.

In order to measure the entanglement given by (11) between two orthogonally polarized modes $a$ and $b$, we can reexpress $\mathcal{I}_{a, b}(\theta)$ as the sum of the noises of circularly polarized modes 1,2 with respect to $a, b$

$$
\mathcal{I}_{a, b}(\theta)=\left\langle\delta X_{1}^{2}(\theta)\right\rangle+\left\langle\delta X_{2}^{2}(\theta)\right\rangle
$$




$$
\text { with } \begin{aligned}
A_{1} & =\frac{1}{\sqrt{2}}\left(A_{a}+A_{b}\right) \\
A_{2} & =\frac{i}{\sqrt{2}}\left(A_{a}-A_{b}\right)
\end{aligned}
$$

As represented in Fig. 3, the modes 1,2 are straightforwardly obtained from the given $a, b$ modes with a half-wave and a quarter-wave plate. They are then mixed with a strong coherent local oscillator (LO) on a polarizing beamsplitter and sent to two balanced homodyne detections. We thus simultaneously measure the spectral noise densities $\left\langle\delta X_{1}^{2}(\theta)\right\rangle$ and $\left\langle\delta X_{2}^{2}(\theta)\right\rangle$ at a given analysis frequency, the sum of which directly gives $\mathcal{I}_{a, b}(\theta)$. This value oscillates when the LO phase is varied in time. Note that, unlike usual detection schemes [7] involving two successive measurements, this method is based on one simultaneous measurement.

\subsection{Case of a linear incident polarization}

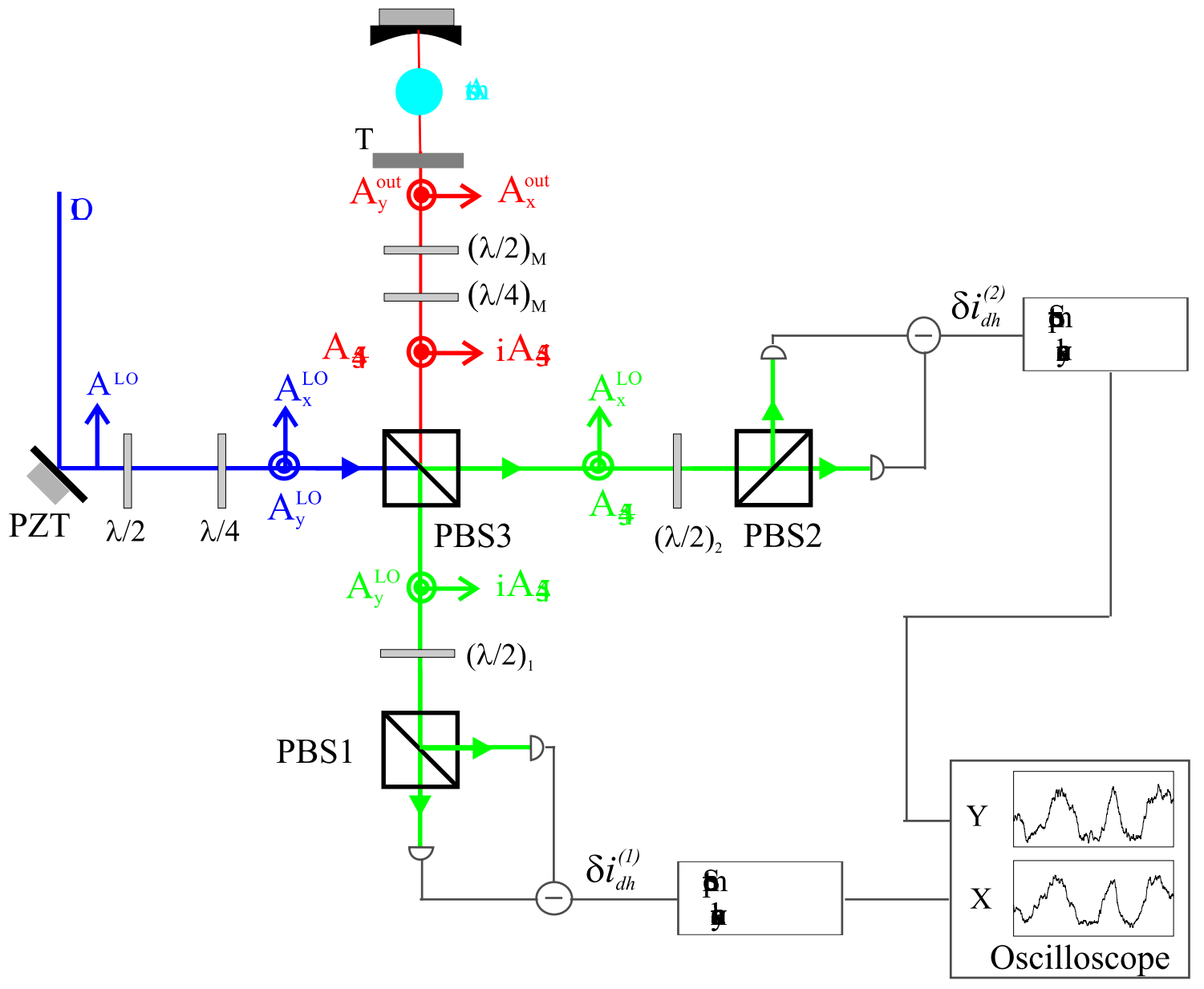

Figure 4. Experimental set-up.

In the system considered in [8], an $x$-polarized beam interacts with a cloud of cold cesium atoms in an optical cavity. The experimental set-up is shown in Fig. 4 . The 
cavity coupling mirror has a transmission coefficient of $10 \%$, the rear mirror is highly reflecting. We probe the atoms with a linearly polarized laser beam detuned by about $50 \mathrm{MHz}$ in the red of the $6 \mathrm{~S}_{1 / 2}, \mathrm{~F}=4$ to $6 \mathrm{P}_{3 / 2}, \mathrm{~F}=5$ transition. The optical power of the probe beam ranges from 5 to $15 \mu \mathrm{W}$. After exiting the cavity, both the mean field mode $A_{x}$ and the orthogonally polarized vacuum mode $A_{y}$ are squeezed for frequencies ranging between 3 and $12 \mathrm{MHz}$. An interpretation of these results [16] can be provided by modelling the complicated $6 \mathrm{~S}_{1 / 2}, \mathrm{~F}=4$ to $6 \mathrm{P}_{3 / 2}, \mathrm{~F}=5$ transition by an X-like four-level atomic structure (Fig. [5).

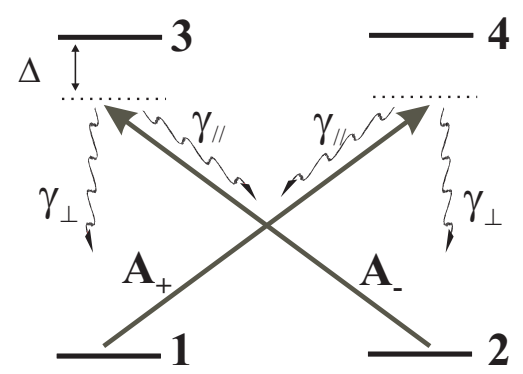

Figure 5. Atomic level structure considered: X-like configuration.

When the two transitions are symmetrically saturated, the atoms behave as a Kerrlike medium for the circular components

$$
A_{ \pm}=\frac{1}{\sqrt{2}}\left(A_{x} \mp i A_{y}\right)
$$

both of which are squeezed [16. Because of the symmetry of the system, they are obviously squeezed for the same quadrature. From the viewpoint of linear polarizations the $x, y$ modes are also squeezed due to cross-Kerr effect, but for orthogonal quadratures [16, 17].

Indeed, from (12), one derives the following expressions

$$
\begin{aligned}
\left\langle\delta A_{x} \delta A_{y}\right\rangle & =\frac{i}{\sqrt{2}}\left(\left\langle\delta A_{+}^{2}\right\rangle-\left\langle\delta A_{-}^{2}\right\rangle+\left\langle\delta A_{+} \delta A_{-}^{\dagger}\right\rangle-\left\langle\delta A_{+}^{\dagger} \delta A_{-}\right\rangle\right)=0 \\
\left\langle\delta A_{x} \delta A_{y}^{\dagger}\right\rangle & \left.=\frac{-i}{\sqrt{2}}\left(\left\langle\delta A_{+} \delta A_{+}^{\dagger}\right\rangle-\left\langle\delta A_{-} \delta A_{-}^{\dagger}\right\rangle+\left\langle\delta A_{+} \delta A_{-}^{\dagger}\right\rangle-\left\langle\delta A_{+}^{\dagger} \delta A_{-}\right\rangle\right) \neq 110\right) \\
\left\langle\delta A_{x}^{2}\right\rangle & =\frac{1}{\sqrt{2}}\left(\left\langle\delta A_{+}^{2}\right\rangle+\left\langle\delta A_{-}^{2}\right\rangle-2\left\langle\delta A_{+} \delta A_{-}\right\rangle\right) \\
\left\langle\delta A_{y}^{2}\right\rangle & =-\frac{1}{\sqrt{2}}\left(\left\langle\delta A_{+}^{2}\right\rangle+\left\langle\delta A_{-}^{2}\right\rangle+2\left\langle\delta A_{+} \delta A_{-}\right\rangle\right)
\end{aligned}
$$

Eqs. (13) 141) show that the $x, y$ modes are completely independent. We then measure $\mathcal{I}_{x, y}(\theta)$ following the previous procedure: $A_{+45}$ and $i A_{-45}$ are sent to the homodyne detections (Fig. (4)), yielding the quantity 


$$
\begin{aligned}
\mathcal{I}_{x, y}(\theta) & =\left\langle\delta X_{+45}^{2}(\theta)\right\rangle+\left\langle\delta X_{i(-45)}^{2}(\theta)\right\rangle \\
\text { with } A_{+45} & =\frac{1}{\sqrt{2}}\left(A_{x}+A_{y}\right) \\
i A_{-45} & =\frac{i}{\sqrt{2}}\left(A_{x}-A_{y}\right)
\end{aligned}
$$

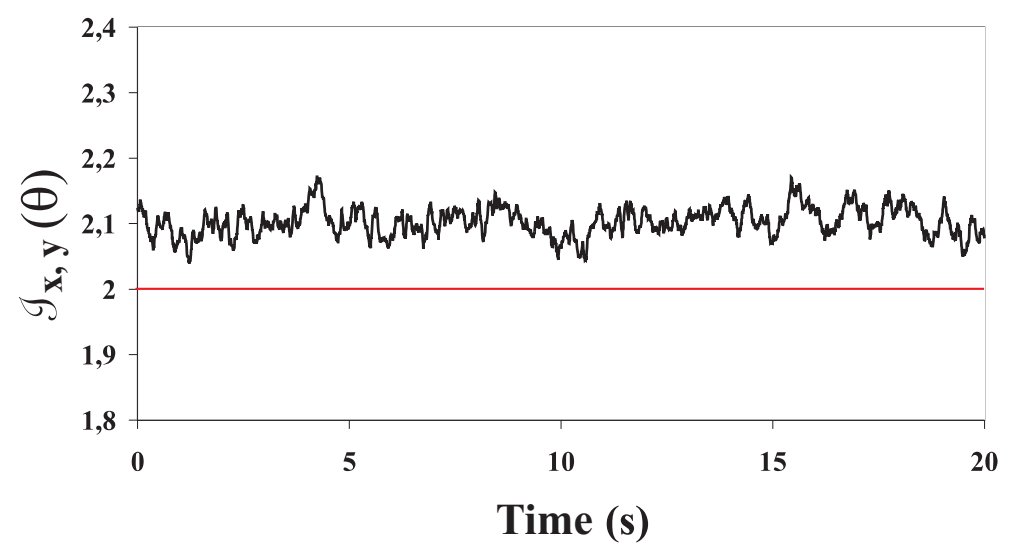

Figure 6. $\mathcal{I}_{x, y}(\theta)$ when $\theta$ is varied in time, at $5 M H z$.

We verify in Fig. [6 that this quantity is effectively independent of $\theta$, ensuring that the $x, y$ modes are "uncorrelated" in the sense of the inseparability criterion.

Bearing in mind that the $u, v$ mode noises must be minimal for the same quadrature, we look at (15,16) to find the relative orientation of the minimal quadratures of the $x, y$ modes. These minimal quadratures are a priori different and depend on the correlation term $\left\langle\delta A_{+} \delta A_{-}\right\rangle$between the circularly polarized modes. Physically, these modes are correlated by optical pumping processes between Zeeman sublevels. However, we place ourselves in the "high frequency" limit for which the analysis frequency (a few $\mathrm{MHz}$ ) is much higher than the optical pumping rate (a few hundreds of $\mathrm{kHz}$ ). The modes $A_{ \pm}$ are then uncorrelated $\left(\left\langle\delta A_{+} \delta A_{-}\right\rangle \simeq 0\right)$, and squeezed for the same quadratures. Using (15, 16) and the fact that

$$
\left\langle\delta X_{\alpha}^{2}\right\rangle=\left\langle\delta A_{\alpha} \delta A_{\alpha}^{\dagger}+\delta A_{\alpha}^{\dagger} \delta A_{\alpha}\right\rangle+2\left\langle\delta A_{\alpha}^{2}\right\rangle \cos 2 \theta, \quad(\alpha=u, v)
$$

we deduce that the $x, y$ modes are squeezed for orthogonal quadratures. These properties were verified both theoretically [16] and experimentally [17] [see also Fig. 7(a)]. In the high frequency limit, one has thus to dephase one mode by $\pi / 2$; we choose for "uncorrelated" basis $A_{u}=A_{x}$ and $A_{v}=i A_{y}$.

The maximally entangled modes are then the modes at $45^{\circ}$ to the $x, y$ basis

$$
\begin{aligned}
& A_{a^{*}}=\frac{1}{\sqrt{2}}\left(A_{u}-i A_{v}\right)=\frac{1}{\sqrt{2}}\left(A_{x}+A_{y}\right) \equiv A_{+45} \\
& A_{b^{*}}=\frac{1}{\sqrt{2}}\left(A_{u}+i A_{v}\right)=\frac{1}{\sqrt{2}}\left(A_{x}-A_{y}\right) \equiv A_{-45}
\end{aligned}
$$


and their entanglement is measured by summing the minimal noises of $A_{x}$ and $i A_{y}$

$$
\mathcal{I}_{+45,-45}=\left\langle\delta X_{x}^{2}\right\rangle_{\min }+\left\langle\delta X_{i y}^{2}\right\rangle_{\min } \simeq 1.9
$$
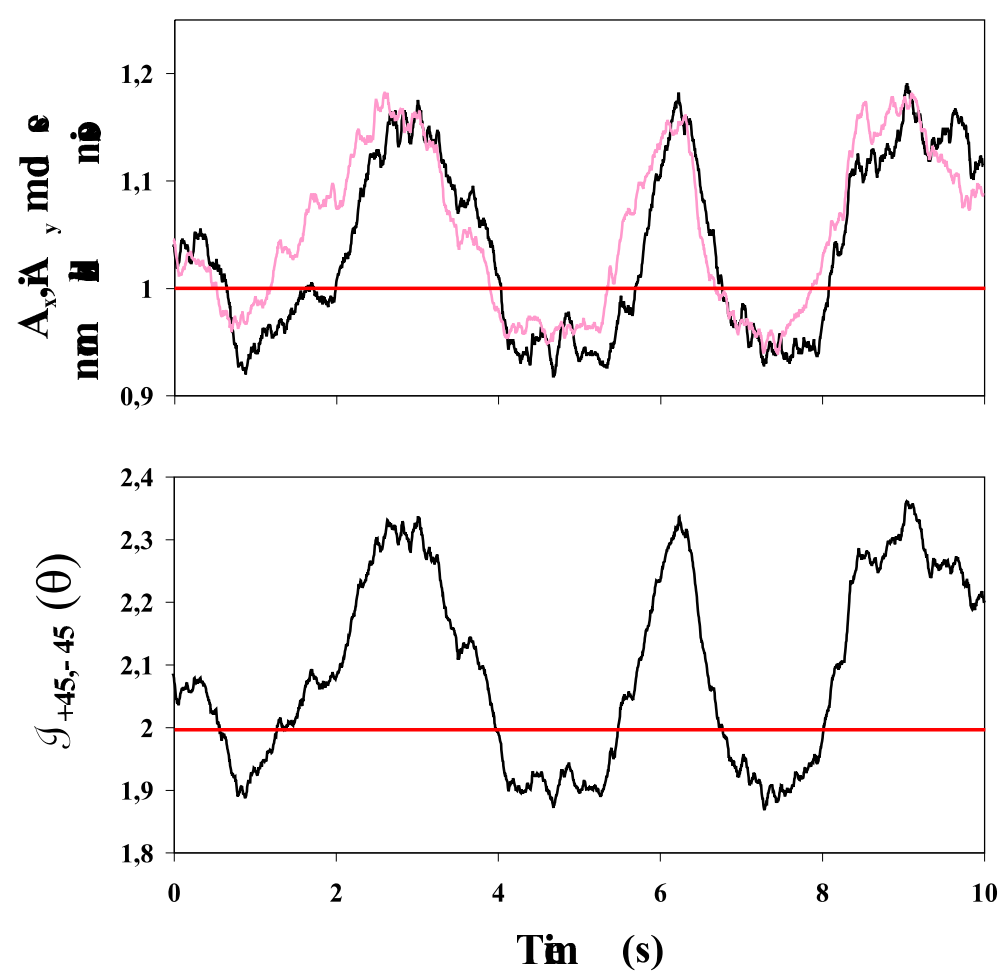

Figure 7. (a) Noise spectra of $A_{x}$ and $i A_{y}$ when the LO phase is varied in time. The analysis frequency is $5 \mathrm{MHz}$. (b) Corresponding value of $\mathcal{I}_{+45,-45}(\theta)$.

The results are reproduced in Fig. 7 for an analysis frequency of $5 M H z$ : the two modes have indeed the same spectrum, and, when summed, the minimal value of $\mathcal{I}_{+45,-45}(\theta)$ is below 2, demonstrating entanglement. Equivalently, one could have set $A_{u}=A_{+}$and $A_{v}=A_{-}$, since these modes are uncorrelated and symmetrical. To obtain the entangled modes, one has to dephase them by $\pi / 2$ and combine them on a beamsplitter, yielding again the $A_{ \pm 45}$ modes

$$
\begin{aligned}
& A_{+45} \equiv \frac{1}{\sqrt{2}}\left(A_{x}+A_{y}\right)=-\frac{e^{i \frac{\pi}{4}}}{\sqrt{2}}\left(A_{+}+i A_{-}\right) \\
& A_{-45} \equiv \frac{1}{\sqrt{2}}\left(A_{x}-A_{y}\right)=-\frac{e^{-i \frac{\pi}{4}}}{\sqrt{2}}\left(A_{+}-i A_{-}\right)
\end{aligned}
$$

Let us now represent these results in the Poincaré sphere. In contrast with the previous Section, we define the Stokes parameters in a more usual fashion from the linearly polarized modes $(x, y)$ basis 

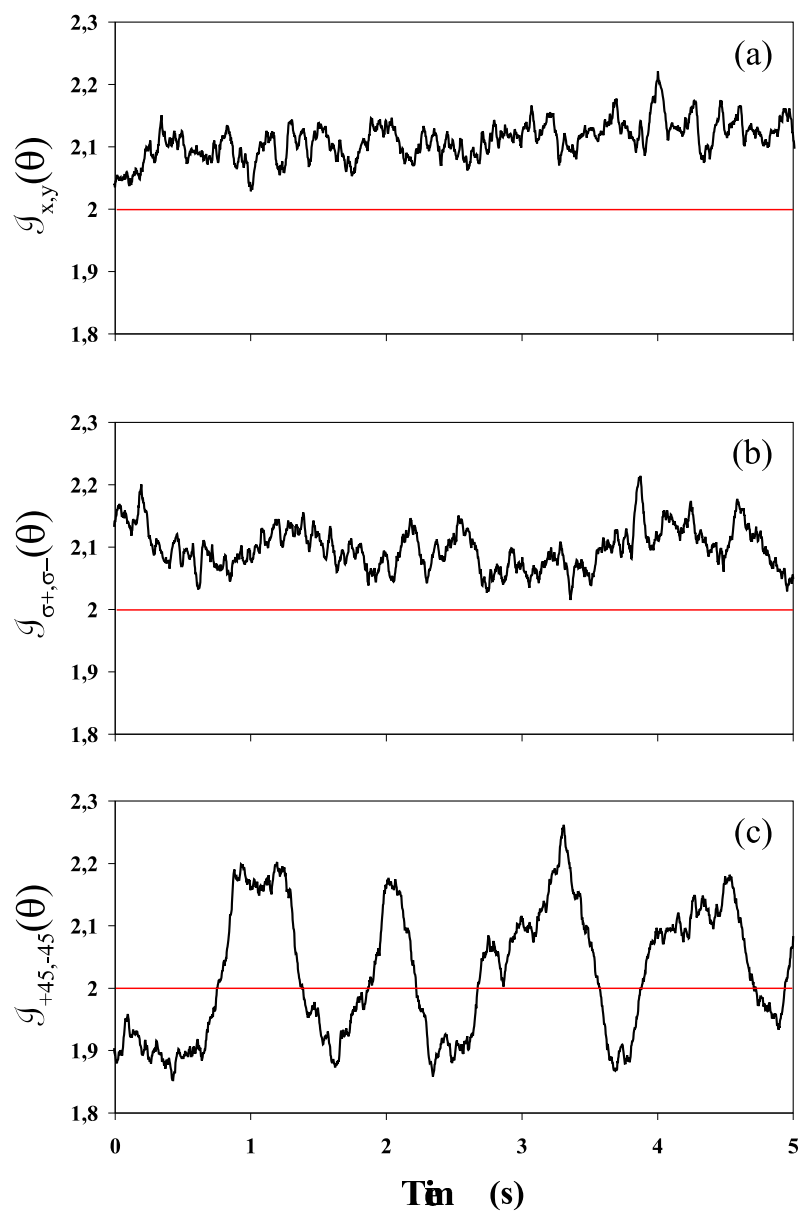

Figure 8. Entanglement for the canonical polarization basis: $(x, y)(\mathrm{a}),\left(\sigma_{+}, \sigma_{-}\right)(\mathrm{b})$, $(+45,-45)(\mathrm{c})$, for an analysis frequency of $5 \mathrm{MHz}$.

$$
\begin{array}{ll}
S_{0}=A_{x}^{*} A_{x}+A_{y}^{*} A_{y} & S_{1}=A_{x}^{*} A_{x}-A_{y}^{*} A_{y} \\
S_{2}=A_{x}^{*} A_{y}+A_{y}^{*} A_{x} & S_{3}=i\left(A_{y}^{*} A_{x}-A_{x}^{*} A_{y}\right)
\end{array}
$$

With the relations $A_{u}=A_{x}, A_{v}=i A_{y}$, it is easy to see that the new Poincaré sphere is obtained from the previous one via a rotation by $\pi / 2$ around the $S_{1}$ axis. If we measure the entanglement in the $(\sigma+, \sigma-)$ basis

$$
\mathcal{I}_{\sigma+, \sigma-}(\theta)=\left\langle\delta X_{x}^{2}(\theta)\right\rangle+\left\langle\delta X_{y}^{2}(\theta)\right\rangle
$$

and represent the results in Fig. 8, one sees that the basis in the $\left(S_{1}, S_{3}\right)$ plane are uncorrelated, as expected, whereas maximal entanglement is found for the $\pm 45^{\circ}$ modes. We also measured the noise spectra of each modes in the three basis and checked that the squeezing is maximal and identical for all modes in the $\left(S_{1}, S_{3}\right)$ plane

$$
\left\langle\delta X_{x}^{2}\right\rangle_{\min }=\left\langle\delta X_{y}^{2}\right\rangle_{\min }=\left\langle\delta X_{\sigma+}^{2}\right\rangle_{\min }=\left\langle\delta X_{\sigma-}^{2}\right\rangle_{\min } \simeq 0.95
$$


and that the entangled modes $A_{ \pm 45}$ have almost isotropic fluctuations in the Fresnel diagram, as well as identical spectra because of the independence of the $x, y$ modes. One can summarize these results in the Poincaré sphere represented in Fig. 9]

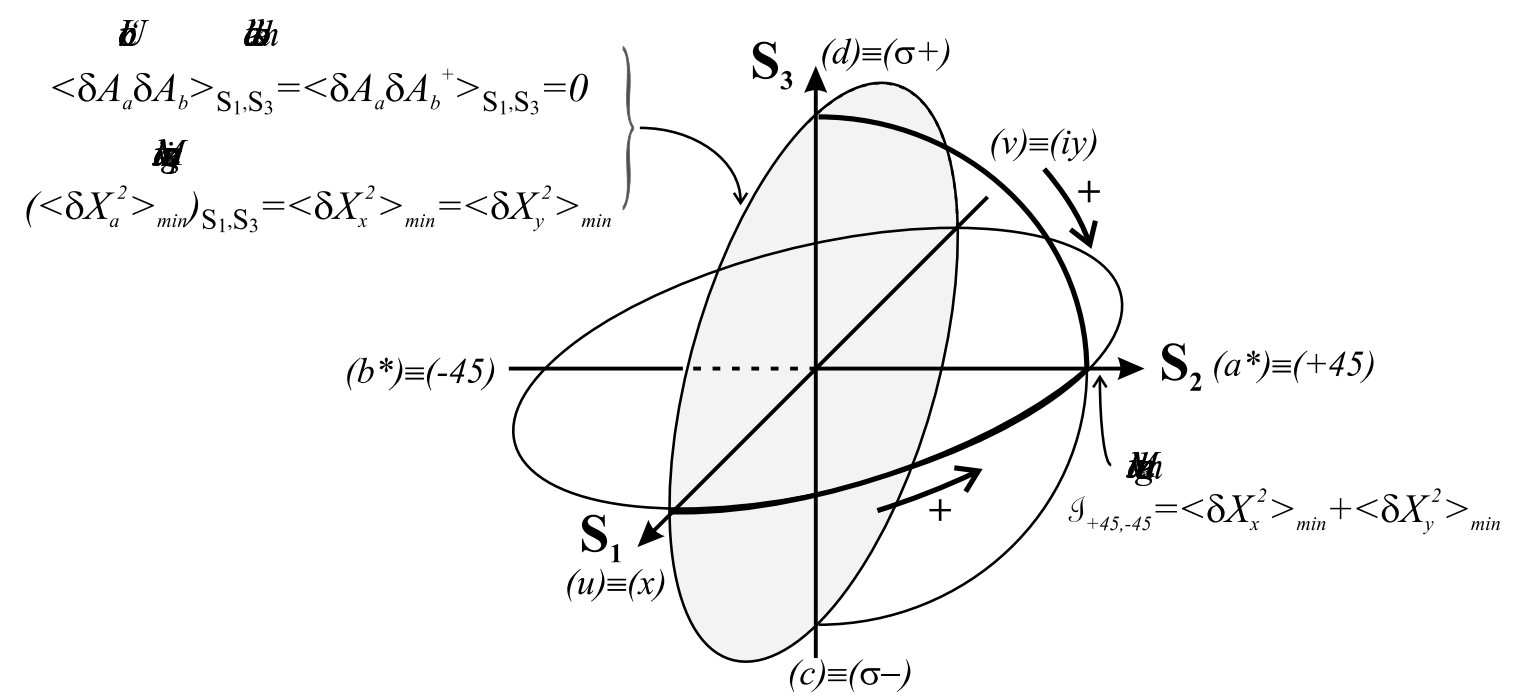

Figure 9. Quantum properties of the beam in the Poincaré sphere at high frequency, in the linear polarization case.

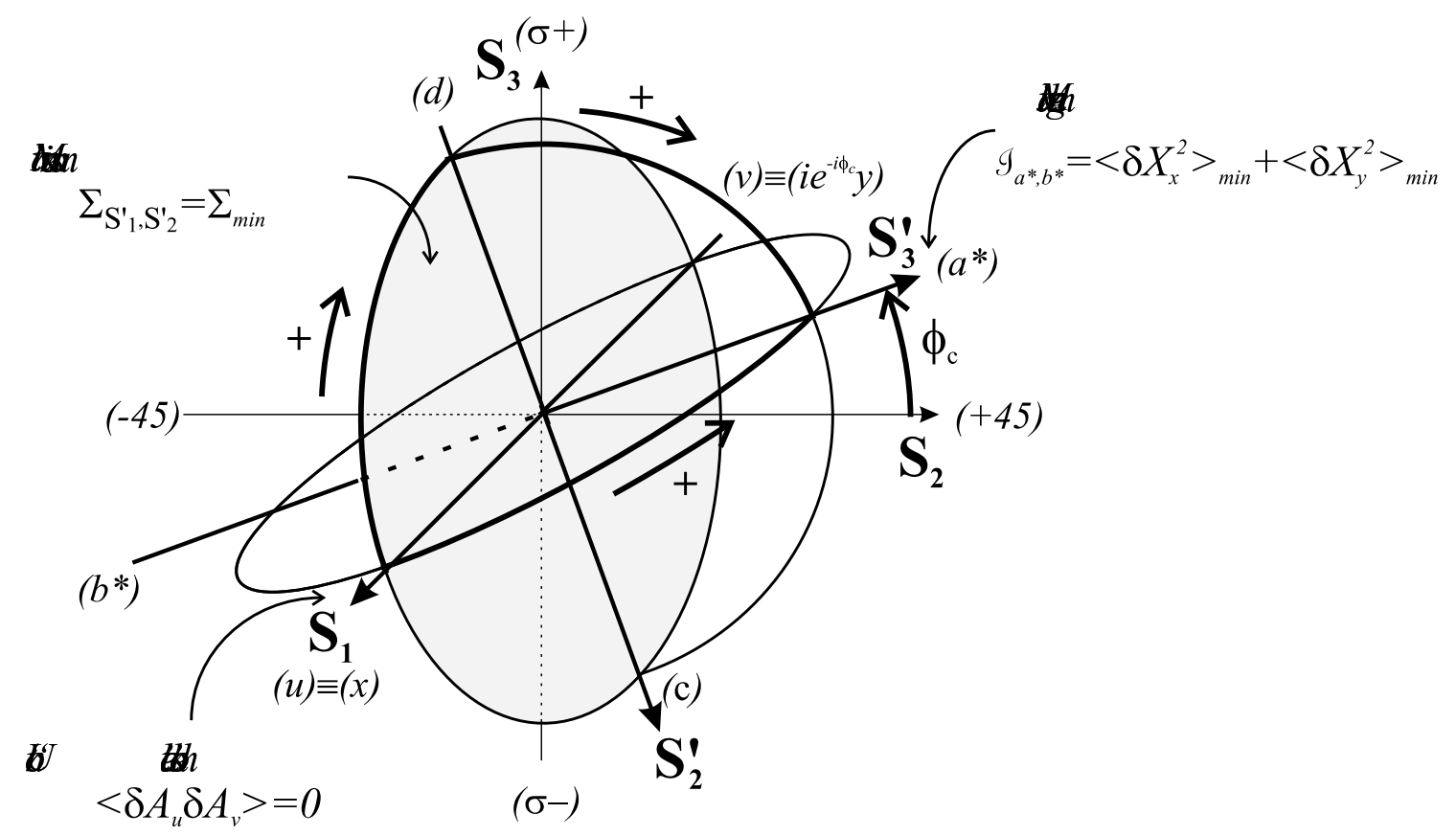

Figure 10. Quantum properties of the beam in the Poincaré sphere at lower frequency, in the linear polarization case.

3.2.1. Frequency dependence The situation is a little bit more complicated at lower frequencies. The correlations between the circularly polarized components must be 
taken into account for frequencies lower than the optical pumping rate. The $\sigma \pm$ modes are still symmetrical and the $x, y$ modes still uncorrelated. However, the minimal noise quadratures are rotated [Eqs. (15, 16)] and the $x, y$ modes are no longer squeezed for orthogonal quadratures. To retrieve the "uncorrelated" basis, one mode must be dephased, say $y$ :

$$
\begin{aligned}
& A_{u}=A_{x} \quad \text { and } \quad A_{v}=i e^{-i \phi_{C}} A_{y} \\
& \text { where } \tan \phi_{C}=\frac{2\left|\left\langle\delta A_{+} \delta A_{-}\right\rangle\right| \sin \left(\phi_{2}-\phi_{1}\right)}{\left\langle\delta A_{+}^{2}\right\rangle+\left\langle\delta A_{-}^{2}\right\rangle+2\left|\left\langle\delta A_{+} \delta A_{-}\right\rangle\right| \cos \left(\phi_{1}-\phi_{2}\right)}
\end{aligned}
$$

In the high frequency limit, $\left|\left\langle\delta A_{+} \delta A_{-}\right\rangle\right| \rightarrow 0$ and, therefore $\phi_{C} \rightarrow 0$ : we retrieve the previous $(u, v)$ basis. This dephasing for the $y$ mode is equivalent to a rotation of the Poincaré sphere by an angle $\phi_{C}$ around the $S_{1}$ axis [Fig. 10]. The maximally entangled modes are then

$$
\begin{aligned}
& A_{a^{*}}=\frac{1}{\sqrt{2}}\left(A_{u}-i A_{v}\right)=\frac{1}{\sqrt{2}}\left(A_{x}+e^{-i \phi_{C}} A_{y}\right) \\
& A_{b^{*}}=\frac{1}{\sqrt{2}}\left(A_{u}+i A_{v}\right)=\frac{1}{\sqrt{2}}\left(A_{x}-e^{-i \phi_{C}} A_{y}\right)
\end{aligned}
$$

and their entanglement is still given by (20)

$$
\mathcal{I}_{a^{*}, b^{*}}=\left\langle\delta X_{x}^{2}\right\rangle_{\min }+\left\langle\delta X_{y}^{2}\right\rangle_{\min }
$$

In Fig. 11 are plotted the $x, y$ modes squeezing versus frequency, as well as $\mathcal{I}_{+45,-45}$ and the optimal entanglement $\mathcal{I}_{a^{*}, b^{*}}$. At low frequency the squeezing improves for the vacuum mode $A_{y}$, but degrades for the mean field mode $x$, so that the entanglement

actually decreases at low frequency. In Fig. 11 we also report the value of $\mathcal{I}_{+45,-45}$. One sees that it equals the optimal entanglement in the high frequency limit, but, for lower frequencies, the two values differ, confirming that the maximally entangled modes are no longer $A_{ \pm 45}$, but given by (21,22).

\subsection{Case of a circular incident polarization}

In this Section, we illustrate the differences between a two squeezed mode system and a single squeezed mode system. We show that the entanglement produced in the latter is qualitatively different from the former, even if the amount of correlations is the same. Whereas the two squeezed modes situation corresponds to the case of a linear polarization, the single squeezed mode situation appears when the polarization is circular. Indeed, our system may exhibit polarization switching: the intracavity polarization may become circular under some conditions [16]. In this case, the atoms only interact with one mode - say $\sigma_{+}$, which may also be squeezed because of Kerr effect. Yet, the situation is very different from the previous one. One can set $A_{u}=A_{+}$and 

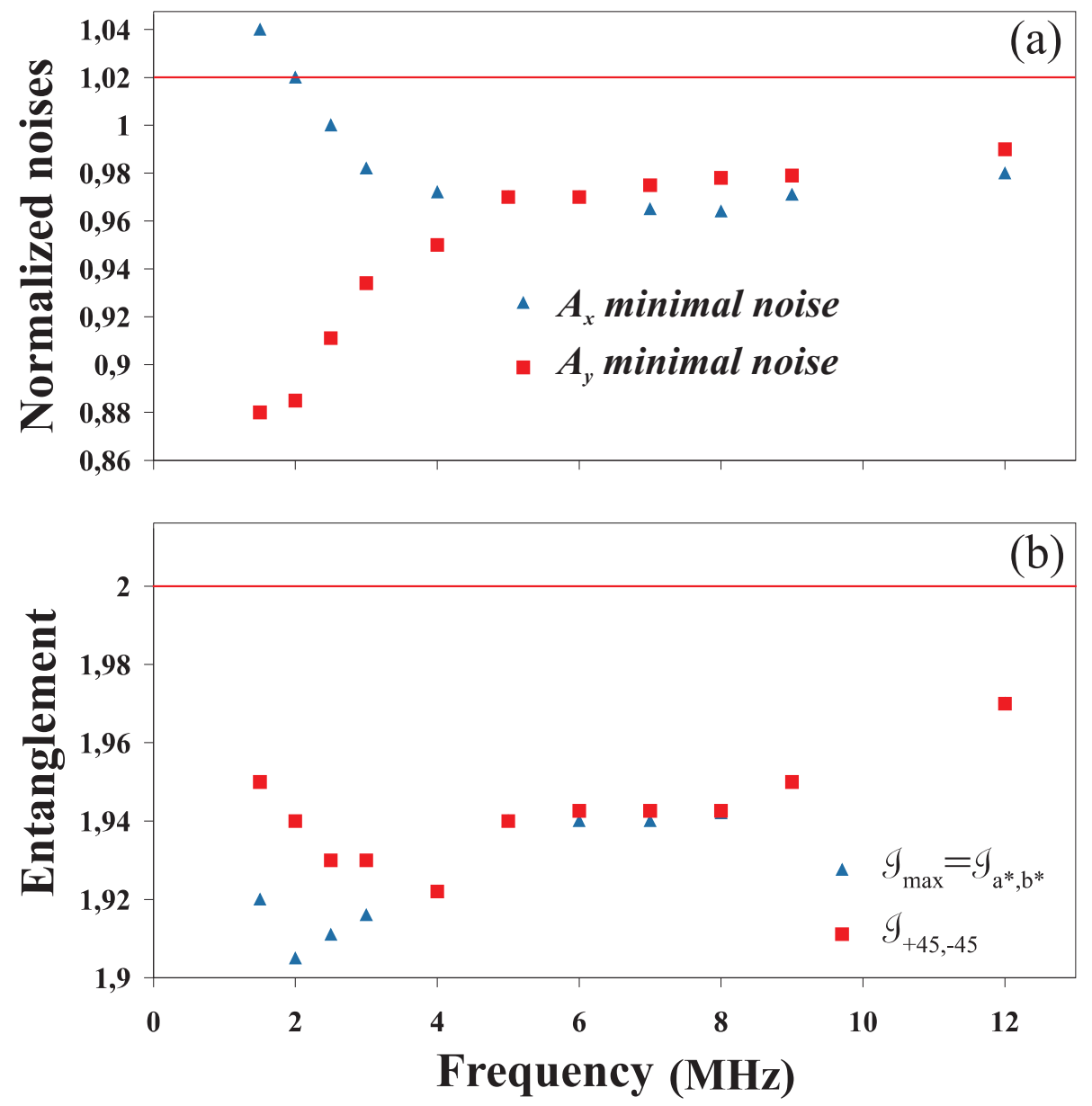

Figure 11. (a) $x, y$ modes minimum noises versus frequency. (b) Entanglement for the $\pm 45^{\circ}$-polarized modes $\left(\mathcal{I}_{+45,-45}\right)$ compared to the maximal entanglement $\left(\mathcal{I}_{a^{*}, b^{*}}\right)$.

$A_{v}=A_{-}$, the former being squeezed, the latter being a coherent vacuum. The vacuum fluctuations are isotropic and, therefore, the properties of the $(u, v)$ basis must remain unchanged when the $v$ mode is dephased. In other words, the Poincaré sphere must be invariant under rotations around $S_{1}$. Moreover, since $\left\langle\delta A_{v}^{2}\right\rangle=0$, the minimal noise sum is uniform on the whole sphere

$$
\Sigma_{\min }=\Sigma_{\max }=\left\langle\delta X_{u}^{2}\right\rangle_{\min }+1
$$

The entanglement is maximal and constant in the $\left(S_{2}, S_{3}\right)$ plane

$$
\mathcal{I}_{S_{2}, S_{3}}=\min _{a, b} \mathcal{I}_{a, b}=\left\langle\delta X_{u}^{2}\right\rangle_{\min }+1
$$

In this plane all modes have identical noise

$$
\left\langle\delta X_{a}^{2}(\theta)\right\rangle=\frac{1}{2}\left(1+\left\langle\delta X_{u}^{2}(\theta)\right\rangle\right)
$$


This result is easily understandable if compared to the transmission of a squeezed beam by a beamsplitter. It is well known that part of the incident beam squeezing is lost to the reflected beam and leaks into the environment.

We measured the noise spectra for all basis. We observed squeezing in the $\sigma_{+}$component, the $\sigma_{-}$component being at the shot noise level. We checked that the spectra of the $x, i y,+45, i(-45)$ modes were all identical and squeezed by half the amount of the $\sigma_{+}$component squeezing, consistently with (23).

Although the $x, y$ modes are both squeezed for orthogonal quadratures, the difference with the previous case is that they are now correlated. We verified this by measuring the entanglement in each basis. The results, displayed in Fig. 12, show that the circular components are indeed uncorrelated, while the $x, y$ modes are entangled, as well as the \pm 45 modes.
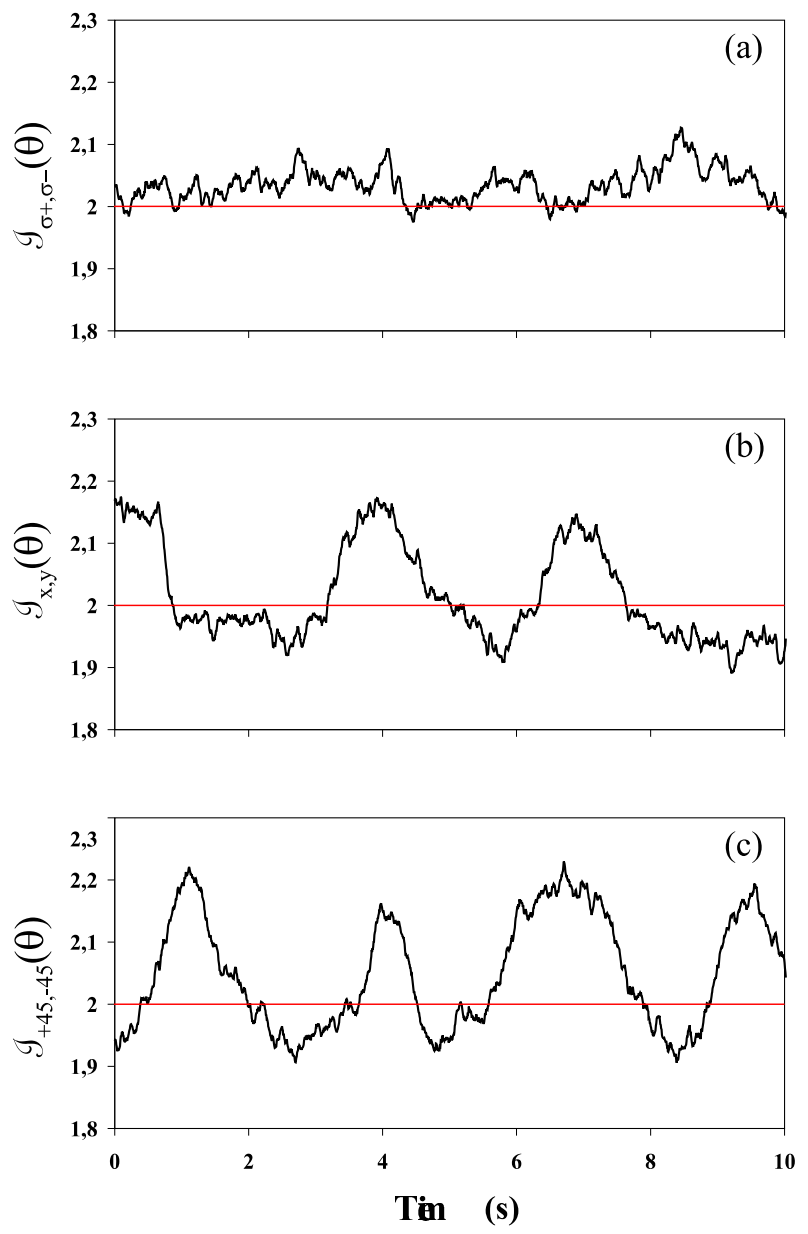

Figure 12. Entanglement in different polarization basis, in the circular polarization case: (a) $\mathcal{I}_{x, y}(\theta)$, (b) $\mathcal{I}_{\sigma+, \sigma-}(\theta)$, (c) $\mathcal{I}_{+45,-45}(\theta)$. 


\section{Polarization entanglement}

Up to now, we have determined the correlation properties of the beam and shown that non-separable states, namely the \pm 45 modes in the high frequency limit, were produced in our system. We show in this Section that the quadrature entanglement demonstrated previously can be mapped into a polarization basis, thus achieving polarization entanglement.

\subsection{Definition and scheme}

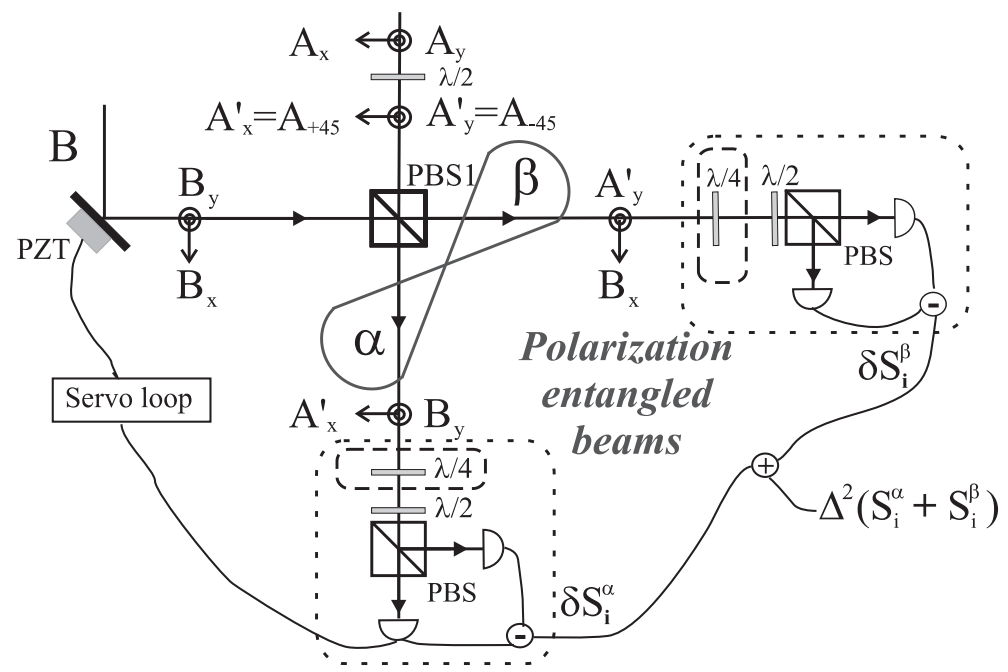

Figure 13. Polarization-entangled beams generation set-up. Using the quarter-wave plates or not allows for measuring either $S_{S_{2}}$ or $S_{S_{3}}$.

Let us consider two spatially separated polarization modes $\alpha$ and $\beta$. To each one we associate a set of Stokes parameters. One can extend the inseparability criterion for two modes to a pair of these operators [8, 10, 7]. Polarization entanglement is achieved when

$$
\mathcal{I}_{\alpha, \beta}^{S}=\frac{1}{2}\left[\left\langle\delta\left(S_{2}^{\alpha}+S_{2}^{\beta}\right)^{2}\right\rangle+\left\langle\delta\left(S_{3}^{\alpha}+S_{3}^{\beta}\right)^{2}\right\rangle\right]<\left|\left\langle\left[S_{2}^{\alpha}, S_{3}^{\alpha}\right]\right\rangle\right|+\left|\left\langle\left[S_{2}^{\beta}, S_{3}^{\beta}\right]\right\rangle\right|
$$

Because of the cyclical commutation relations between the Stokes operators, the criterion now depends on the polarization state of the beams (here, $\left\langle S_{1}^{\alpha}\right\rangle$ and $\left\langle S_{1}^{\beta}\right\rangle$ ). In our case, we use two quadrature entangled modes $a$ and $b$, mix them on a polarizing beamsplitter with an intense coherent beam $B$, polarized at $45^{\circ}$ [Fig. 13]. The resulting beams, $\alpha$ and $\beta$, are composed with modes $A_{a}$ and $B_{y}$, and $A_{b}$ and $B_{x}$ respectively. The Stokes parameters are

$$
\begin{array}{ll}
S_{1}^{\alpha}=A_{a}^{\dagger} A_{a}-B_{y}^{\dagger} B_{y} & S_{1}^{\beta}=B_{x}^{\dagger} B_{x}-A_{b}^{\dagger} A_{b} \\
S_{2}^{\alpha}=A_{a} B_{y}^{\dagger}+A_{a}^{\dagger} B_{y} & S_{2}^{\beta}=A_{b}^{\dagger} B_{x}+A_{b} B_{x}^{\dagger} \\
S_{3}^{\alpha}=i\left(A_{a} B_{y}^{\dagger}-A_{a}^{\dagger} B_{y}\right) & S_{3}^{\beta}=i\left(A_{b}^{\dagger} B_{x}-A_{b} B_{x}^{\dagger}\right)
\end{array}
$$


Denoting by $\alpha_{B}, \alpha_{a}, \alpha_{b}$ and $\theta_{B}$ the field amplitudes and the B-field phase, and assuming that the B-field is much more intense than the A-field $\left(\alpha_{B} \gg \alpha_{a}\right.$, $\left.\alpha_{b}\right)$, the two beams are orthogonally polarized: $\left\langle S_{1}^{\alpha}\right\rangle=-\left\langle S_{1}^{\beta}\right\rangle=-\alpha_{B}^{2}$. The polarization entanglement condition (24) then reads

$$
\mathcal{I}_{\alpha, \beta}^{S}<2 \alpha_{B}^{2}
$$

On the other hand, the Stokes parameters fluctuations are proportional to the $a, b$ modes quadratures

$$
\begin{array}{llrl}
\delta S_{2}^{\alpha} & =\alpha_{B} \delta X_{a}\left(\theta_{B}\right), & \delta S_{2}^{\beta} & =\alpha_{B} \delta X_{b}\left(\theta_{B}\right) \\
\delta S_{3}^{\alpha} & =-\alpha_{B} \delta Y_{a}\left(\theta_{B}\right), & \delta S_{3}^{\beta} & =\alpha_{B} \delta Y_{b}\left(\theta_{B}\right)
\end{array}
$$

The inseparability criterion is thus directly related to the entanglement between the $a, b$ modes

$$
\mathcal{I}_{\alpha, \beta}^{S}=\alpha_{B}^{2} \mathcal{I}_{a, b}\left(\theta_{B}\right)
$$

If one locks the phase $\theta_{B}$ in order to obtain $\mathcal{I}_{a, b}\left(\theta_{B}\right)=\min _{\theta} \mathcal{I}_{a, b}(\theta) \equiv \mathcal{I}_{a, b}<2$, the beams are then polarization entangled. The $S_{2}$ and $S_{3}$ Stokes parameters can be measured using the right combination of plates and beamsplitter [10].

\subsection{Experimental results}

We have shown that the maximally entangled modes were the \pm 45 modes at the output of the cavity. We thus insert after the cavity a half-wave plate to set $A_{a}=A_{+45}$ and $A_{b}=A_{-45}$. We then lock the phase $\theta_{B}$ and measure

$$
S_{S_{2}}=\left\langle\left(\delta X_{x}\left(\theta_{B}\right)\right)^{2}\right\rangle \quad, \quad S_{S_{3}}=\left\langle\left(\delta Y_{y}\left(\theta_{B}\right)\right)^{2}\right\rangle
$$

The results are shown in Fig. 14 and we observe

$$
\begin{aligned}
& S_{S_{2}} \equiv \frac{1}{2 \alpha_{B}^{2}}\left\langle\delta\left(S_{2}^{\alpha}+S_{2}^{\beta}\right)^{2}\right\rangle \simeq 0.96<1 \\
& S_{S_{3}} \equiv \frac{1}{2 \alpha_{B}^{2}}\left\langle\delta\left(S_{3}^{\alpha}+S_{3}^{\beta}\right)^{2}\right\rangle \simeq 0.96<1
\end{aligned}
$$

so that

$$
\frac{\mathcal{I}_{\alpha, \beta}^{S}}{\alpha_{B}^{2}}=S_{S_{2}}+S_{S_{3}}=\mathcal{I}_{+45, i(-45)} \simeq 1.92<2
$$

This value is consistent with the quadrature entanglement measurement (20). 

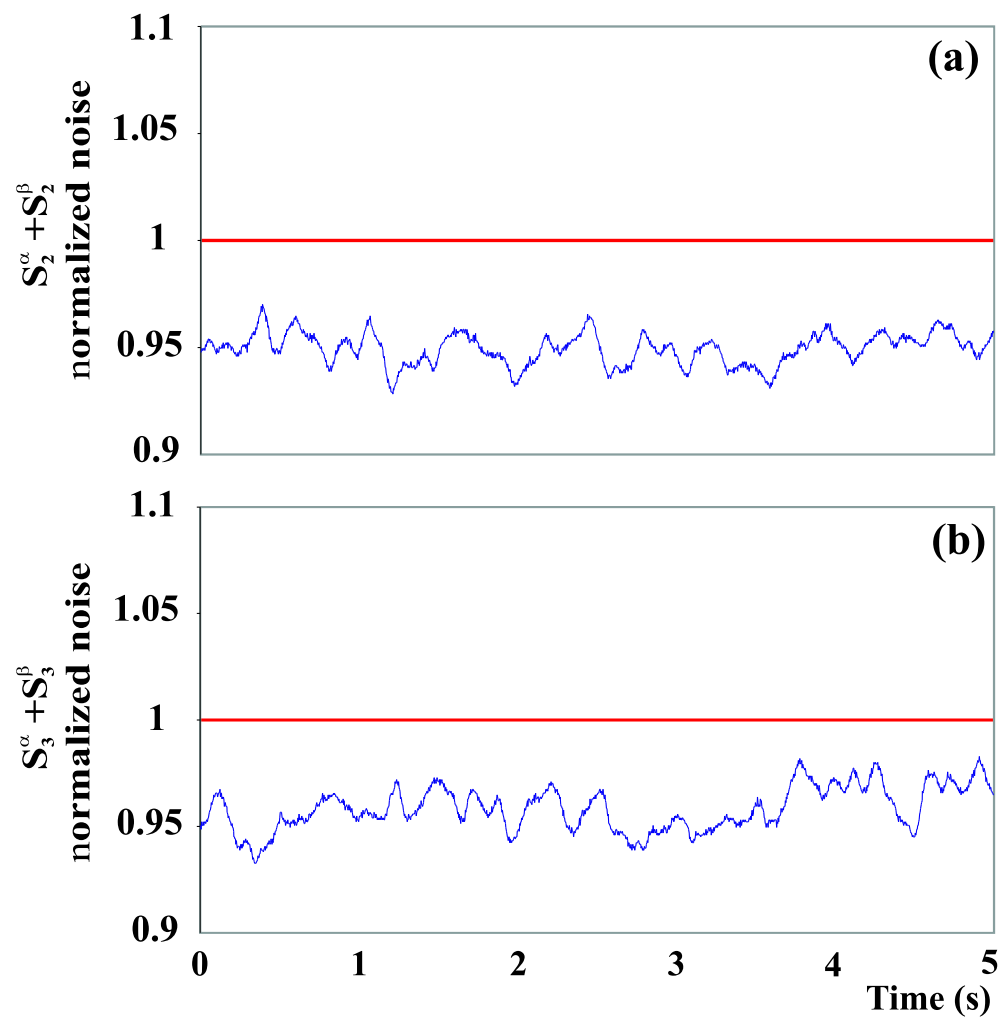

Figure 14. Normalized noises of $S_{2}^{\alpha}+S_{2}^{\beta}$ (a) and $S_{3}^{\alpha}+S_{3}^{\beta}$ (b), when the phase $\theta_{B}$ is locked.

\section{Conclusion}

Using the non-linearity of cold atoms, we have generated a quadrature-entangled beam. The maximally entangled polarization modes have been found following a general method to characterize entanglement in a two mode system. We have stressed the equivalence between our scheme and other continuous variable entanglement experiments in which the correlations are created via the mixing of independent beams. Moreover, a simple interpretation of the quantum properties of such a system was given in the Poincar sphere. To demonstrate the entanglement we have performed a direct measurement of the inseparability criterion [2] using two homodyne detections. We have then achieved polarization entanglement by mixing our quadrature entangled beam with an intense coherent field. Experimental evidence of this entanglement was given by the direct measurement of the Stokes operators noises for each beam.

\section{Appendix A. Uncorrelated basis existence}

Starting from a "correlated" polarization basis $(a, b)\left(\left\langle\delta A_{a} \delta A_{b}\right\rangle \neq 0\right)$, we prove the existence of the "uncorrelated" basis $(u, v)$, such that

$$
\left\langle\delta A_{u} \delta A_{v}\right\rangle=0
$$


Using the following decomposition

$$
\begin{aligned}
& A_{u}=\cos \Phi A_{a}-\sin \Phi e^{i \omega} A_{b} \\
& A_{v}=\sin \Phi A_{a}+\cos \Phi e^{i \omega} A_{b}
\end{aligned}
$$

with

$$
\begin{aligned}
& e^{i \omega}=\frac{\mathcal{M}}{|\mathcal{M}|}, \quad \cos 2 \Phi=\frac{\left|\left\langle\delta A_{a}^{2}\right\rangle\right|^{2}-\left|\left\langle\delta A_{b}^{2}\right\rangle\right|^{2}}{\mathcal{N}}, \quad \sin 2 \Phi=\frac{-2|\mathcal{M}|}{\mathcal{N}} \\
& \text { where } \quad \mathcal{M}=\left\langle\delta A_{a}^{2}\right\rangle\left\langle\delta A_{a} \delta A_{b}\right\rangle^{*}+\left\langle\delta A_{b}^{2}\right\rangle^{*}\left\langle\delta A_{a} \delta A_{b}\right\rangle \\
& \text { and } \quad \mathcal{N}=\sqrt{4|\mathcal{M}|^{2}+\left[\left|\left\langle\delta A_{a}^{2}\right\rangle\right|^{2}-\left|\left\langle\delta A_{b}^{2}\right\rangle\right|^{2}\right]^{2}}
\end{aligned}
$$

it is straightforward to see that the $u, v$ modes thus defined satisfy (A.1).

\section{Appendix B. Correlations in the Poincaré sphere}

We give a brief demonstration of the general properties about the Poincaré sphere enunciated in Sec. 2.2. Given the Poincaré sphere defined from the uncorrelated basis $(u, v)$, we calculate the entanglement and squeezing in different basis. We recall that the entanglement $\mathcal{I}_{a, b}$ between modes $a$ and $b$ only depends on the correlation term $\left|\left\langle\delta A_{a} \delta A_{b}\right\rangle\right|$, whereas the noise sum $\Sigma_{a, b}$ depends on the quantity $\left|\left\langle\delta A_{a}^{2}\right\rangle\right|^{2}+\left|\left\langle\delta A_{b}^{2}\right\rangle\right|^{2}$. For a given polarization basis (4/5), one has

$$
\begin{aligned}
& \left|\left\langle\delta A_{a}^{2}\right\rangle\right|^{2}=\beta^{4}\left\langle\delta A_{u}^{2}\right\rangle^{2}+\alpha^{4}\left\langle\delta A_{v}^{2}\right\rangle^{2}+2 \alpha^{2} \beta^{2}\left\langle\delta A_{u}^{2}\right\rangle\left\langle\delta A_{v}^{2}\right\rangle \cos 2 \phi \\
& \left|\left\langle\delta A_{b}^{2}\right\rangle\right|^{2}=\alpha^{4}\left\langle\delta A_{u}^{2}\right\rangle^{2}+\beta^{4}\left\langle\delta A_{v}^{2}\right\rangle^{2}+2 \alpha^{2} \beta^{2}\left\langle\delta A_{u}^{2}\right\rangle\left\langle\delta A_{v}^{2}\right\rangle \cos 2 \phi
\end{aligned}
$$

For $\alpha$ and $\beta$ fixed, $\left|\left\langle\delta A_{a}^{2}\right\rangle\right|$ and $\left|\left\langle\delta A_{b}^{2}\right\rangle\right|$ are maximal pour $\phi=0[\pi]$

$$
\begin{aligned}
& \left|\left\langle\delta A_{a}^{2}\right\rangle\right|_{\max }=\beta^{2}\left\langle\delta A_{u}^{2}\right\rangle+\alpha^{2}\left\langle\delta A_{v}^{2}\right\rangle \quad \text { and }\left|\left\langle\delta A_{b}^{2}\right\rangle\right|_{\max }=\alpha^{2}\left\langle\delta A_{u}^{2}\right\rangle+\beta^{2}\left\langle\delta A_{v}^{2}\right\rangle \\
& \Rightarrow \quad\left(\left|\left\langle\delta A_{a}^{2}\right\rangle\right|+\left|\left\langle\delta A_{b}^{2}\right\rangle\right|\right)_{\phi=0}=\max _{a, b}\left[\left|\left\langle\delta A_{a}^{2}\right\rangle\right|+\left|\left\langle\delta A_{b}^{2}\right\rangle\right|\right]=\left\langle\delta A_{u}^{2}\right\rangle+\left\langle\delta A_{v}^{2}\right\rangle
\end{aligned}
$$

The noise sum is thus minimal for all the linearly polarized modes with respect to $u, v$, i.e. in the plane $\left(S_{1}^{\prime}, S_{2}^{\prime}\right)$

$$
\Sigma_{S_{1}^{\prime}, S_{2}^{\prime}}=\min _{a, b} \Sigma_{a, b}=\left\langle\delta X_{u}^{2}\right\rangle_{\min }+\left\langle\delta X_{v}^{2}\right\rangle_{\min } \equiv \Sigma_{\min }
$$

If the ellipticity increases $(\phi \neq 0)$, the noise sum increases, as can be seen from (B.1,B.2). In the meridional plane $\left(S_{2}^{\prime}, S_{3}^{\prime}\right)$ the $a, b$ modes satisfy $(\alpha=\beta=1 / \sqrt{2})$

$$
\left(\left|\left\langle\delta A_{a}^{2}\right\rangle\right|^{2}\right)_{S_{2}^{\prime}, S_{3}^{\prime}}=\left(\left|\left\langle\delta A_{b}^{2}\right\rangle\right|^{2}\right)_{S_{2}^{\prime}, S_{3}^{\prime}}=\frac{1}{4}\left\{\left\langle\delta A_{u}^{2}\right\rangle^{2}+\left\langle\delta A_{v}^{2}\right\rangle^{2}+2\left\langle\delta A_{u}^{2}\right\rangle\left\langle\delta A_{v}^{2}\right\rangle \cos (2 \Phi)\right\}
$$


Entanglement and squeezing in a two-mode system

This quantity is minimal for the circularly polarized modes $a^{*}, b^{*}(\phi=\pi / 2)$ :

$$
\left|\left\langle\delta A_{a^{*}}^{2}\right\rangle\right|+\left|\left\langle\delta A_{b^{*}}^{2}\right\rangle\right|=\left|\left\langle\delta A_{u}^{2}\right\rangle-\left\langle\delta A_{v}^{2}\right\rangle\right|=\min _{a, b}\left\{\left|\left\langle\delta A_{a}^{2}\right\rangle\right|+\left|\left\langle\delta A_{b}^{2}\right\rangle\right|\right\}
$$

The noise sum then equals its maximal value $\Sigma_{\max }$. Assuming $\left\langle\delta A_{u}^{2}\right\rangle \geq\left\langle\delta A_{v}^{2}\right\rangle$, this value reads

$$
\Sigma_{a^{*}, b^{*}}=\left\langle\delta X_{a^{*}}^{2}\right\rangle_{\min }+\left\langle\delta X_{b^{*}}^{2}\right\rangle_{\min }=\left\langle\delta X_{u}^{2}\right\rangle_{\min }+\left\langle\delta X_{v}^{2}\right\rangle_{\max } \equiv \Sigma_{\max }
$$

More generally, one has

$$
\Sigma_{\max }=\min \left\{\left\langle\delta X_{u}^{2}\right\rangle_{\min }+\left\langle\delta X_{v}^{2}\right\rangle_{\max },\left\langle\delta X_{u}^{2}\right\rangle_{\max }+\left\langle\delta X_{v}^{2}\right\rangle_{\min }\right\}
$$

Let us now consider the entanglement. Optimal entanglement is obtained by construction for modes $a^{*}, b^{*}$

$$
\mathcal{I}_{a^{*}, b^{*}}=\min _{a, b} \mathcal{I}_{a, b}=\left\langle\delta X_{u}^{2}\right\rangle_{\text {min }}+\left\langle\delta X_{v}^{2}\right\rangle_{\text {min }}=\Sigma_{\text {min }}
$$

The entanglement decreases with the ellipticity. For the linearly polarized modes $(\phi=0)$, Eq. (6) yields

$$
\left|\left\langle\delta A_{a} \delta A_{b}\right\rangle\right|=\alpha \beta\left|\left\langle\delta A_{u}^{2}\right\rangle-\left\langle\delta A_{v}^{2}\right\rangle\right|
$$

$\mathcal{I}_{a, b}$ reaches its maximal value for the $u, v$ modes

$$
\mathcal{I}_{u, v}=\frac{1}{2}\left\{\left\langle\delta X_{u}^{2}\right\rangle_{\min }+\left\langle\delta X_{u}^{2}\right\rangle_{\max }+\left\langle\delta X_{v}^{2}\right\rangle_{\min }+\left\langle\delta X_{v}^{2}\right\rangle_{\max }\right\} \equiv \max _{a, b} \mathcal{I}_{a, b}
$$

However, $\mathcal{I}_{a, b}$ is not constant in the equatorial plane; it is minimal $\left(\mathcal{I}_{c, d}=\Sigma_{\text {max }}\right)$ for the $45^{\circ}$-polarized modes $(\alpha=\beta=1 / \sqrt{2})$, denoted by $c, d$.

Last, we would like to point out that optimizing the squeezing sum of two modes is not equivalent to optimizing the squeezing for one mode only. Finding the maximally squeezed mode is not trivial, since the $u, v$ modes are not a priori independent. No condition holds on $\left\langle\delta A_{u} \delta A_{v}^{\dagger}\right\rangle$. The noise of one quadrature of mode $a$ is given by

$$
\left\langle\delta X_{a}^{2}(\theta)\right\rangle=\beta^{2}\left\langle\delta X_{u}^{2}(\theta)\right\rangle+\alpha^{2}\left\langle\delta X_{v}^{2}(\theta-\phi)\right\rangle-2 \alpha \beta\left\langle\delta X_{u}(\theta) \delta X_{v}(\theta-\phi)\right\rangle
$$

The correlation term can be written as

$$
\begin{aligned}
\left\langle\delta X_{u}(\theta) \delta X_{v}(\theta-\phi)\right\rangle & =\left\langle\delta A_{u} \delta A_{v}^{\dagger}\right\rangle e^{-i \phi}+\left\langle\delta A_{u}^{\dagger} \delta A_{v}\right\rangle e^{+i \phi} \\
& =2 \cos \left(\phi_{C}-\phi\right)\left|\left\langle\delta A_{u} \delta A_{v}^{\dagger}\right\rangle\right|
\end{aligned}
$$


with $\phi_{C}$ the phase of $\left\langle\delta A_{u} \delta A_{v}^{\dagger}\right\rangle$. The complex general solution takes a simple form if we assume that $\left\langle\delta A_{u} \delta A_{v}^{\dagger}\right\rangle$ is a real positive number $\left(\phi_{C}=0\right)$. The optimal value is then reached for $\phi=0$ and one finally gets

$$
\begin{aligned}
\min _{a}\left\{\left\langle\delta X_{a}^{2}\right\rangle_{\min }\right\}= & \frac{1}{2}\left\{\left\langle\delta X_{u}^{2}\right\rangle_{\min }+\left\langle\delta X_{v}^{2}\right\rangle_{\min }\right. \\
& \left.-\sqrt{\left(\left\langle\delta X_{u}^{2}\right\rangle_{\min }-\left\langle\delta X_{v}^{2}\right\rangle_{\min }\right)^{2}+16\left\langle\delta A_{u} \delta A_{v}^{\dagger}\right\rangle^{2}}\right\}
\end{aligned}
$$

It is therefore possible to obtain a better squeezing on one mode than that of $u, v$. Note that, if $\left\langle\delta A_{u} \delta A_{v}^{\dagger}\right\rangle=0$, the $u, v$ modes are independent and, as in our experiments, the best squeezing is that of one of the $u, v$ modes.

[1] M.D. Reid, Phys. Rev. A 37, 4792 (1988).

[2] L.M. Duan, G. Giedke, J.I. Cirac, P. Zoller, Phys. Rev. Lett. 84, 2722 (2000); R. Simon, Phys. Rev. Lett. 84, 2726 (2000).

[3] A. Furusawa, J. Sorensen, S. Braunstein, C. Fuchs, H.J. Kimble, E.S. Polzik, Science 282, 706 (1998).

[4] J. Hald, J.L. Sorensen, C. Schori, E.S. Polzik, Phys. Rev. Lett. 83, 1319 (1999).

[5] Y. Zhang, H. Wang, X. Li, J. Jing, C. Xie, K. Peng, Phys. Rev. A 62, 023813 (2000).

[6] C. Silberhorn, P.K. Lam, O. Weiß, F. König, N. Korolkova, G. Leuchs, Phys. Rev. Lett. 86, 4267 (2001).

[7] W.P. Bowen, N. Treps, R. Schnabel, P.K. Lam, Phys. Rev. Lett. 89, 253601 (2002); W.P. Bowen, N. Treps, R. Schnabel, T.C. Ralph, P.K. Lam, J. Opt. B Quantum Semiclassical 5, S467 (2003).

[8] V. Josse, A. Dantan, A. Bramati, M. Pinard, E. Giacobino, quant-ph/0306147

[9] B. Julsgaard, A. Koshekin, E.S. Polzik, Nature 413, 400 (2001).

[10] N. Korolkova, G. Leuchs, R. Loudon, T.C. Ralph, C. Silberhorn, Phys. Rev. A 65, 052306 (2002); N. Korolkova, Ch. Silberhorn, O. Glöckl, S. Lorentz, Ch. Marquardt, G. Leuchs, Eur. Phys. J. D. 18, 229 (2002).

[11] O. Glöckl, J. Heersink, N. Korolkova, G. Leuchs, S. Lorenz, quant-ph/0302083

[12] W.P. Bowen, N. Treps, B.C Buchler, R. Schnabel, T.C. Ralph, H.A. Bachor, T. Symul, P.K. Lam, Phys. Rev. A 67, 032302 (2003).

[13] X. Li, Q. Pan, J. Jing, J. Zhang, C. Xie, K. Peng, Phys. Rev. Lett. 88, 047904 (2002).

[14] O. Glöckl, S. Lorenz, C. Marquardt, J. Heersink, M. Brownnutt, C. Silberhorn, Q. Pan, P. van Loock, N. Korolkova, G. Leuchs, Phys. Rev A 68, 012319 (2003).

[15] W.P. Bowen, R. Schnabel, P.K. Lam, T.C. Ralph, Phys. Rev. Lett. 90, 043601 (2003).

[16] V. Josse, A. Dantan, A. Bramati, M. Pinard, E. Giacobino, J. Opt. B Quantum Semiclassical 5, S1 (2003).

[17] V. Josse, A. Dantan, L. Vernac, A. Bramati, M. Pinard, E. Giacobino, Phys. Rev. Lett. 90, 103601 (2003).

[18] A. Einstein, B. Podolsky, N. Rosen, Phys. Rev. 47, 777 (1935).

[19] L. Longchambon, J. Laurat, T. Coudreau, C. Fabre, in preparation.

[20] S. Huard, Polarization of light, John Wiley and Sons, New York (1997), p. 26. 